\title{
THREE-DIMENSIONAL MODELS OF GEOENVIRONMENTAL PARAMETERS
}

\author{
Shestakov V.V \\ Tomsk Polytechnic University \\ Tomsk, Russian federation \\ e-mail:valeriy.shestakov@inbox.ru \\ Stepanov D.Yu. \\ Tomsk Polytechnic University \\ Tomsk, Russian federation \\ e-mail: sdu@am.tpu.ru
}

\author{
Sysolyatina G.A. \\ LTD Scientific Analytic Center "Nedra" \\ Tomsk, Russian federation \\ e-mail:nedraGA@mail.tomsknet.ru
}

\begin{abstract}
The article proposes the modified Kriging method for synthesis of three-dimensional model of geoenvironmental parameters based on data of land and borehole seismic survey.
\end{abstract}

Key words - geostatistical modeling, seismic survey, Kriging, geophysical borehole studies.

Irregular grid-based spatial data modeling is currently used in many spheres of activities. Indeed, in geophysics, geoenvironmental parameters (porosity, permeability, etc.) are modeled based on the results of geophysical borehole studies (GBS) by various methods [1]. In the general case, such modeling should resolve itself into solution to a problem of three-dimensional interpolation whose solution may be based on the known deterministic methods (linear interpolators, inverse square method, basis function methods, etc.) [2]. Such approach provides good results in the field of significant concentration of basic data (boreholes). However, in case of offset distance from boreholes of hundreds of meters, the accuracy of deterministic methods decreases to unacceptable values.

Statistical methods of interpolation in this situation allow for enhancing the accuracy of prognostic predictions. The geostatistical modeling and, in particular, Kriging method based on the analysis of spatial distribution and correlation relationships of the known values of interpolated field are the most popular [3]. The disadvantages of Kriging method comprise the constraint of obligatory homogeneity and object stationarity and the hypothesis of unboundness of the domain for the values of interpolated filed which generate physically unreal results. In case of sparse grid of prestack data, Kriging models are extremely rough and hardly differ from the deterministic ones.

To eliminate the latter disadvantage, this work proposes to modify Kriging method through the analysis of prognostic parameters and data of land seismic survey $(2 \mathrm{D}$ or $3 \mathrm{D}$ common depth point seismic (CDPS)). Combined use of land and borehole seismic survey data is based on the fact that CDPS has significantly more detailed grid of observations and the changes of a seismic attribute indirectly contain information on the changes of a prognostic geoenvironmental parameter [4]. In this case, the statement of a geostatistical problem for the evaluation of spatial data may be changed as follows:

- Let $N$ boreholes are drilled at points $p_{i}\left(x_{i}, y_{i}\right.$, forming the irregular grid.

- In each borehole, the measurements of a prognostic parameter are performed and are presented as geophysical well logging (GWL) curve $f_{i}(t), i=1, . ., N$. Let us consider the obtained GWL curves a sample of prognostic parameter field $f_{i}(t)=F\left(x_{i}, y_{i}, t\right)$.

- In the study zone, land seismic survey is performed setting the seismic attribute traces for the set of points (amplitude, energy, frequency energy ratios, etc.).

- Only in case of performing 3D CDPS, this set forms the regular grid. Therefore, we will further believe that the reconstructed parameter is defined only at a point with coordinates $(x, y)$, for which a seismic attribute is known: $S(x, y, t)$

In accordance with Kriging equation, the desired interpolation is sought in the form of the best linear combination of the known $f_{i}(t)$ :

$$
\hat{F}(x, y, t)=\sum_{i=1}^{N} w_{i}(x, y, t) f_{i}(t),
$$

where $w_{i}(x, y, t)$ is the weight function of $i$-th borehole. As shown in [3], unbiasedness of estimate (1) in fixed arguments $x, y$ and $t$ is achieved upon condition

$$
\sum_{i=1}^{N} w_{i}(x, y, t)=1,
$$

and selection of the weight functions is determined by minimization of error variance:

$$
D[F(x, y, t)-\hat{F}(x, y, t)] \rightarrow \min .
$$


In Kriging, the hypothesis on homogeneity and stationarity $F(x, y, t)$ is used in the wide sense (covariance function does not depend on coordinates, but only on a distance between the points and on time deviation) [5]. This hypothesis leads to task simplification and use of variograms instead of covariance functions. Modeling of variograms is performed mostly by linear methods. All mentioned does not allow Kriging method to fully take into account spatial changes of inhomogeneous geological environment.

By discarding the idea of homogeneity and stationarity of the field $F(x, y, t)$, one has to abandon the modeling of variograms and to replace it with the model based on the properties of the field $S(x, y, t)$. If there is a statistical association between $S(x, y, t)$ and $F(x, y, t)$ i.e. changes of covariance properties of inhomogeneous and nonstationary field $S(x, y, t)$ carry information on the changes of covariance properties $F(x, y, t)$, then unknown weight functions may be found from the equation of interpolation of seismic attribute:

$$
\widehat{S}(x, y, t)=\sum_{i=1}^{N} w_{i}(x, y, t) s_{i}(t)
$$

where $s_{i}(t)=S\left(x_{i}, y_{i}, t\right)$ is a seismic attribute trace in $i$-th point of a borehole. Constraint of consistency (3) rearranges to the following form:

$$
D\left[S(x, y, t)-\sum_{i=1}^{N} w_{i}(x, y, t) s_{i}(t)\right] \rightarrow \min .
$$

Let us view the solution of assigned task at an arbitrary point in space $p_{0}=\left(x_{0}, y_{0}\right)$ :

$$
\begin{aligned}
& S\left(x_{0}, y_{0}, t\right)=S_{0}(t) \\
& w_{i}^{0}(t)=w_{i}\left(x_{0}, y_{0}, t\right) .
\end{aligned}
$$

Considering that, at point $p_{0}$, estimate (4) is unbiased, i.e. $M\left[S_{0}(t)-\sum_{i=1}^{N} w_{i}^{0}(t) s_{i}(t)\right]=0$, the equation (5) can be rewritten in the following form:

$$
\begin{gathered}
D\left[S_{0}(t)-\sum_{i=1}^{N} w_{i}^{0}(t) s_{i}(t)\right]= \\
=M\left[\left(S_{0}(t)-\sum_{i=1}^{N} w_{i}^{0}(t) s_{i}(t)\right)^{2}\right]= \\
=M\left[S_{0}(t)^{2}\right]-2 M\left[S_{0}(t) \sum_{i=1}^{N} w_{i}^{0}(t) s_{i}(t)\right]+ \\
+M\left[\sum_{i=1}^{N} w_{i}^{0}(t) s_{i}(t) \sum_{i=1}^{N} w_{j}^{0}(t) s_{j}(t)\right]=
\end{gathered}
$$

$$
\begin{gathered}
=\psi_{0}^{2}(t)-2 \sum_{i=1}^{N} w_{i}^{0}(t) M\left[S_{0}(t) s_{i}(t)\right]+ \\
+\sum_{i=1}^{N} \sum_{j=1}^{N} w_{i}^{0}(t) w_{j}^{0}(t) M\left[s_{i}(t) s_{j}(t)\right]= \\
=\psi_{0}^{2}(t)-2 \sum_{i=1}^{N} w_{i}^{0}(t) C_{0 i}(t)+ \\
+\sum_{i=1}^{N} \sum_{j=1}^{N} w_{i}^{0}(t) w_{j}^{0}(t) C_{i j}(t)
\end{gathered}
$$

where $\psi_{0}^{2}(t)$ is mean value at point $\left(x_{0}, y_{0}\right)$; $C_{0 i}(t)=C_{0 i}(t, t)=M\left[S_{0}(t) s_{i}(t)\right]$ is covariance function of seismic attribute $S(x, y, t)$ at point $p_{0}$ and at point $p_{i}$; $c_{i j}(t)=c_{i j}(t, t)=M\left[S_{i}(t) s_{i}(t)\right]$ is covariance function of seismic attribute $S(x, y, t)$ at points of $i$-th and $j$-th boreholes with coordinates $\left(x_{i}, y_{i}\right)$ and $\left(x_{j}, y_{j}\right)$, respectively.

Now, after differentiating the equation (8) with respect to each desired weight function $w_{i}^{0}(t)$,we receive a system of linear equations of the form:

$$
C(t) \cdot W^{0}(t)=C^{0}(t),
$$

where covariance matrix of seismic attribute at points of

boreholes $-C(t)=\left(\begin{array}{cccc}c_{11}(t) & c_{12}(t) & \ldots & c_{1 n}(t) \\ c_{21}(t) & c_{22}(t) & \cdots & c_{2 n}(t) \\ \vdots & \vdots & & \vdots \\ c_{n 1}(t) & c_{n 2}(t) & \cdots & c_{n n}(t)\end{array}\right) ;$ matrix of

weight functions $-W^{0}(t)=\left(\begin{array}{c}w_{1}^{0}(t) \\ w_{2}^{0}(t) \\ \vdots \\ w_{N}^{0}(t)\end{array}\right)$; covariance matrix of seismic attribute at prognostic point $p_{0}$ and at points of boreholes $-C_{0}(t)=\left(\begin{array}{c}C_{01}(t) \\ C_{02}(t) \\ \vdots \\ C_{0 N}(t)\end{array}\right)$.

Solution of this system will be the values of weight functions $w_{i}^{0}(t)$ where the fulfillment of the condition (5) is achieved. For convenience of further calculations, the left and the right parts of the system (9) are normalized to dimensionless form through division of each equation by $c_{i i}(t)$, and, the equation is added for taking into account the condition (2) by the method of Lagrange multipliers:

$$
w_{1}^{0}(t)+w_{2}^{0}(t)+\cdots+w_{N}^{0}(t)=1 .
$$

As a result, the system will have the form 
where $R(t)=\left(\begin{array}{ccccc}1 & r_{12}(t) & \cdots & r_{1 n}(t) & 1 \\ r_{12}(t) & 1 & \cdots & r_{2 n}(t) & 1 \\ \vdots & \vdots & & \vdots & \vdots \\ r_{1 n}(t) & r_{2 n}(t) & \cdots & 1 & 1 \\ 1 & 1 & \cdots & 1 & 0\end{array}\right)$ is normalized covariance matrix of seismic attribute at borehole points; $R^{0}(t)=\left(\begin{array}{c}r_{01}(t) \\ r_{02}(t) \\ \vdots \\ r_{0 N}(t) \\ 1\end{array}\right)$ is normalized covariance matrix of seismic attribute at prognostic point $p_{0}$ and at points of borehole; $Q(t)=\left(\begin{array}{c}w_{1}^{0}(t) \\ w_{2}^{0}(t) \\ \vdots \\ w_{N}^{0}(t) \\ \alpha(t)\end{array}\right)$ is the matrix of weight functions.

For the fixed time point $t$, system (11) becomes a system of linear algebraic equations (SLAE) that, in case of nonsingularity of the $R(t)$, is solved by any of the known methods (matrix method, Gauss' method, iteration method, etc.). For the real geological environments, seismic traces carry information on vertical inhomogeneity of rocks and, in the general case, are nonstationary. For the analysis of similar processes, let us introduce the hypothesis of their local stationarity. With this supposition, the normalized covariance functions can be calculated through time averaging in running window.

To eliminate the above-mentioned problem of unboundness of the domain for values of the interpolated field, let us introduce additional constraint for the values of the weight coefficients:

$$
0 \leq w_{i}^{0}, i=\overline{1, N}
$$

It is clear that, in case of simultaneous satisfying the conditions (12) and (2), the values of the interpolated field will not exceed the limits defined by prestack data. However, due to the fact that the constraint (12) represents inequation, it is impossible to add it to the system (11). Therefore, the following algorithm is proposed for its implicit adherence:

1. SLAE (11) is solved by any known method.

2. If there are negative values among the elements $Q$, then the corresponding lines and columns in the matrices $R$, $R^{0}$ and $Q$ are removed; after that, algorithm is invoked again from step 1 .

3. In case if all elements $Q$ are nonnegative, then obtained solution is accepted and the algorithm is discontinued.
Due to the fat that the accuracy of model development directly depends on the quality of aprior CDPS and GBS data, the step of data acquisition and preparation is important. This process can be divided into several successive steps:

1. Borehole GBS data acquisition. Due to inhomogeneity of geologic environment, each borehole is unique. The absence of one of boreholes can attenuate the model reliability. Due to this, it is important to initially acquire information on the maximum number of boreholes.

2. CDPS data selection. It is necessary to identify which attribute possesses the covariance properties similar to the properties of the prognostic field. For this, selection of traces for various seismic attributes close to each borehole is performed; after that, covariance relationships between the traces and the GBS curves are determined. Among the seismic attributes, the one with covariance matrix close to the covariance matrix of the prognostic parameter is selected.

3. Formation of a set of the linearly independent traces of seismic attribute. In cases of the closely-spaced boreholes or small variability of seismic attribute, the system (11) will have a singular matrix. This stage allows for avoiding it and for eliminating information excessive for the algorithm.

Primary testing of the method was performed with math modeling MATLAB environment with satisfactory results providing a background for further method implementation by means of programming language $\mathrm{C \#}$ in the form of software application. Testing of this program was done with real data from Tomsk region oil fields. Materials of 3D CDPS and GBS of six boreholes were used for modeling. Prognostic parameter was alpha-SP measured by the method of spontaneous potential logging of rocks. Covariance analysis showed that the attribute "amplitude" after special processing corresponded to this parameter the most (Figure 1).

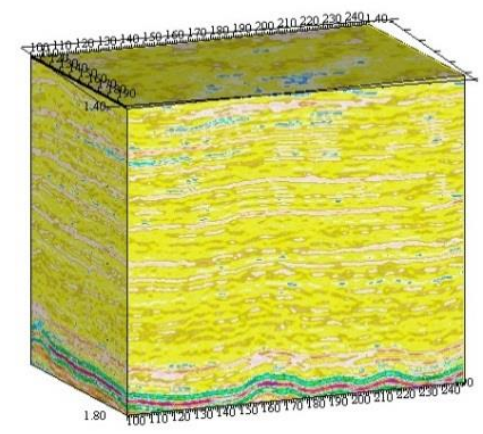

Figure 1. 3D CDPS data.

Generated 3D model for distribution of the parameter of oil filed alpha-SP allows for the evaluation of sedimentary section in areal option (Figure 2 and 3). Based on this model, zones with increased reservoir properties and clayish reservoir-seal rocks (alpha-SP <0.3) are clearly identified (alpha-SP >0.4). This prediction of selected geological bodies plays an 
important role in the field development as well as in solving the tasks for further exploration of the reservoir. The weight functions obtained in the process of the modeling represent valuable information and may be used for solving of subsequent tasks, for example, in performing seismic facies analysis, determination of formation productivity field, etc. (Figure 4).

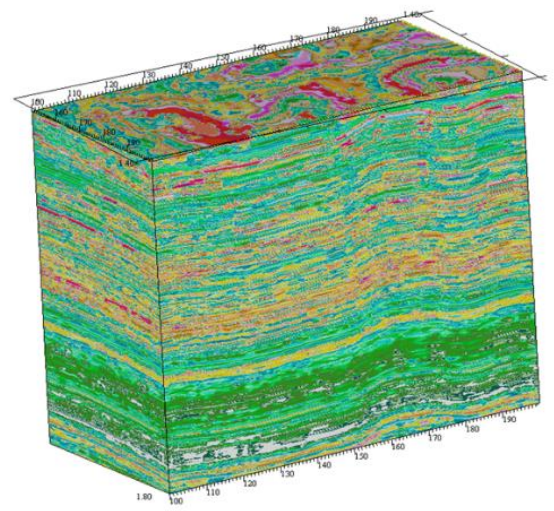

Figure 2. Model of alpha-SP parameter.

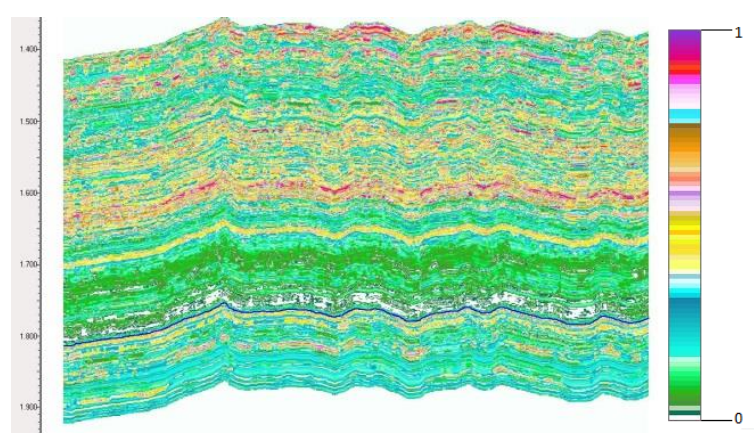

Figure 3. Vertical section of three-dimensional model of alpha-SP (Crossline 125) and its color gradation.

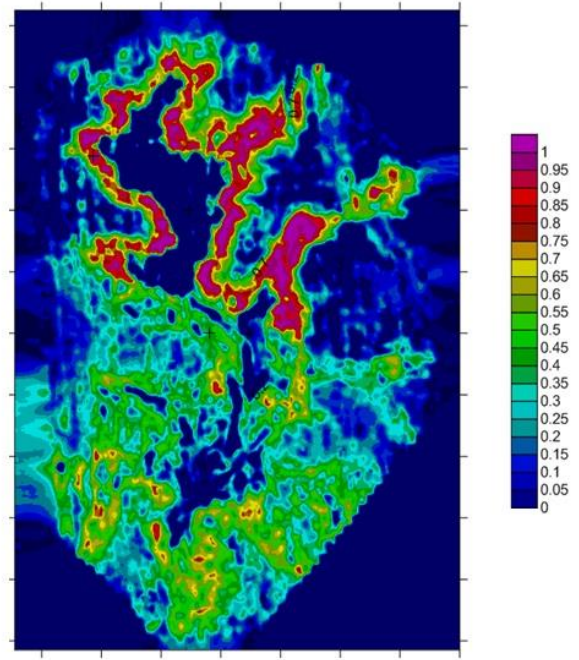

Figure 4. Horizontal section of the weight function for one of boreholes.

The outlined new geostatistical method, though proposed for building the three-dimensional models for parameters of geological environments through integration of CDPS and GBS data, may be generalized for solving similar tasks in other spheres.

[1] V.N. Koskov. Geophysical studies of boreholes. // Perm, Perm State Technical University. $2004-122$ pp.

[2] V.V. Dementiev, E.A. Savelieva. Geostatistics, theory, and practice. Moscow, Nauka. $2010-328$ pp.

[3] Zh. Materon. The basics of applied geostatistics / Izhevsk Printing and Publishing: Izhevsk. $2009-460$ pp.

[4] A.K. Urupov. The basics of three-dimentional seismic survey / Moscow, Oil and Gas. 2004 - 584 pp.

[5] V.N. Kutrunov, M.V. Dmitrievskikh. Analogue of interpolation Krigning method without geostatistical justification // Vestnik TumGU N3. 2001. 208-216 pp. 